\title{
Analytical Mechanics of Chemical Reactions. III. Natural Collision Coordinates*
}

\author{
R. A. Marcus \\ Noyes Chemical Laboratory, University of Illinois, Urbana, Illinois 61801
}

(Received 13 March 1967)

\begin{abstract}
The coordinates of earlier papers of this series are extended from linear collisions to reactions in three dimensions. Termed "natural collision coordinates," they have a unique property of passing smoothly from those suited to reactants to those suited to products. Potential applications to bimolecular reactions are described.
\end{abstract}

The analytical mechanics (quantum and classical) of chemically reactive linear collisions were treated in earlier papers of this series. ${ }^{1,2}$ The main features of the treatment were (1) a set of coordinates which passed smoothly from those suited to reactants, through those suited to "activated complexes," to those suited to products, (2) a zeroth-order solution which was "vibrationally adiabatic,"3 and (3) a calculation of nonadiabatic corrections.

In the present paper this coordinate system is extended to reactions in three dimensions. These coordinates are closely related to the actual vibrations, the free and hindered rotations, and the translations of the system at each stage of the reaction. As Parts I and II illustrate, the corresponding equations of motion are more amenable to approximate analytical solution than are those expressed in conventional coordinates.

The collision-theory literature contains many approximate procedures and it is best to separate them from the actual elaborate definition of the new coordinates. Approximate solutions are given in subsequent papers of this series. Several intuitive applications were given elsewhere. ${ }^{4}$

\section{COORDINATES}

In the case of bimolecular reactions, we consider first the case of a single reaction path,

$$
\mathrm{AB}+\mathrm{C} \rightarrow \mathrm{A}+\mathrm{BC}
$$

* Acknowledgment is made to the donors of the Petroleum Research Fund, administered by the American Chemical Society, for partial support of this research. This research was also supported by a grant from the National Science Foundation at the University of Illinois and by a National Aeronautics and Space Administration Grant NsG-275-62 while the author was a Visiting Professor at the Theoretical Chemistry Institute of the University of Wisconsin.

1 R. A. Marcus, J. Chem. Phys. 45, 4493 (1966), Part I.

2 R. A. Marcus, J. Chem. Phys. 45, 4500 (1966), Part II. Typographical corrections are (a) in Eq. (17) and preceding sentence replace $\left(1+\kappa x_{0}\right)$ by $\left(1+\kappa x_{0}\right)^{2}$, (b) after $\mu\left(1+\kappa x_{0}\right)^{2}$ in that sentence add "on Curve C", and (c) replace $\omega^{2}$ by $\mu \omega^{2}$ in sentence after Eq. (21).

${ }_{3}$ This term is used here to describe reactions which are adiabatic with respect to all vibrational-rotational motions, i.e., to all motions but the one along the reaction coordinate s. A relevance of adiabaticity of internal motions to activated complex theory has been discussed by J. O. Hirschfelder and E. Wigner, J. Chem. Phys. 7, 616 (1939); M. A. Eliason and J. O. Hirschfelder, ibid. 30,

1426 (1959) ; L. Hofacker, Z. Naturforsch. 18a, 607 (1963); R. A. Marcus, J. Chem. Phys. 43, 1598 (1965).

${ }_{4}$ R. A. Marcus, Discussions Faraday Soc. 44, 7 (1967), and discussion remarks. and later include a second path,

$$
\mathrm{AB}+\mathrm{C} \rightarrow \mathrm{AC}+\mathrm{B} \text {. }
$$

The coordinates of the $\alpha$ th atom in the center-of-mass system are denoted by ${ }^{0} x_{\alpha},{ }^{0} y_{\alpha},{ }^{0} z_{\alpha}$. Six scaled relative coordinates in the center of mass are defined via $(3), 5,6$

$$
\begin{aligned}
{ }^{0} x & =\left({ }^{0} x_{\mathrm{B}}-{ }^{0} x_{\mathrm{A}}\right) c, \\
{ }^{0} X & =\left\{{ }^{0} x_{c}-\left[\left(m_{\mathrm{A}}^{0} x_{\mathrm{A}}+m_{\mathrm{B}}^{0} x_{\mathrm{B}}\right) /\left(m_{\mathrm{A}}+m_{\mathrm{B}}\right)\right]\right\} c^{-1},
\end{aligned}
$$

where $c$ is $\left[m_{\mathrm{A}} m_{\mathrm{B}}\left(m_{\mathrm{A}}+m_{\mathrm{B}}+m_{\mathrm{C}}\right) / m_{\mathrm{C}}\left(m_{\mathrm{A}}+m_{\mathrm{B}}\right)^{2}\right]^{1 / 4}$ and where the other components $\left({ }^{0} y\right.$, etc.) are obtained by replacing ${ }^{0} x_{\alpha}$ by ${ }^{0} y_{\alpha}$, etc. The kinetic energy in the center-of-mass system is $T$,

$$
T=\frac{1}{2} \mu\left({ }^{0} \dot{x}^{2}+{ }^{0} \dot{y}^{2}+{ }^{0} \dot{z}^{2}+{ }^{0} \dot{X}^{2}+{ }^{0} \dot{Y}^{2}+{ }^{0} \dot{Z}^{2}\right),
$$

where $\mu$ is $\left[m_{\mathrm{A}} m_{\mathrm{B}} m_{\mathrm{C}} /\left(m_{\mathrm{A}}+m_{\mathrm{B}}+m_{\mathrm{C}}\right)\right]^{1 / 2}$.

The above six space-fixed coordinates are transformed into body-fixed coordinates via Eulerian angles $(\theta, \phi, \chi)$ in a standard way, ${ }^{7}$ as in Fig. 1

$$
\left(\begin{array}{c}
{ }^{0} x \\
{ }^{0} y \\
{ }^{z} z
\end{array}\right)=\mathrm{S}_{\chi^{\theta \phi}}{ }^{T}\left(\begin{array}{l}
x \\
y \\
z
\end{array}\right),
$$

with ${ }^{0} X,{ }^{0} Y,{ }^{0} Z$ being related to $X, Y, Z$ by the same equation and $\mathbf{S}_{X \theta \phi}{ }^{T}$ being the rotation matrix ${ }^{7}$; the elements of $\mathbf{S}$ are direction cosines.

The body-fixed plane $x_{\alpha}=0$ is chosen as the instantaneous plane of the three atoms. Thus,

$$
x=X=0 \text {. }
$$

${ }^{5}$ Compare S. Glasstone, K. J. Laidler, and H. Eyring, The Theory of Rate Processes (McGraw-Hill Book Co., New York, 1941), Chap. 3; F. T. Smith, J. Chem. Phys. 31, 1352 (1959); Phys. Rev. 120, 1058 (1960).

'These coordinates are related to six others for the products, ${ }^{0} x_{p}=-\left({ }^{0} x_{\mathrm{C}}-{ }^{0} x_{\mathbf{B}}\right) c_{p},{ }^{0} X_{p}$

$$
=\left\{\left[\left(m_{\mathrm{B}}{ }^{0} x_{\mathrm{B}}+m_{\mathrm{C}}{ }^{0} x_{\mathrm{C}}\right) /\left(m_{\mathrm{A}}+m_{\mathrm{B}}\right)\right]-{ }^{0} x_{\mathrm{A}}\right\} c_{p}{ }^{-1},
$$

by a simple rotation in Fig. 3 through the angle $\zeta_{\infty}$. Here, $c_{p}$ equals $\left[m_{\mathrm{B}} m_{\mathrm{C}}\left(m_{\mathrm{A}}+m_{\mathrm{B}}+m_{\mathrm{C}}\right) / m_{\mathrm{A}}\left(m_{\mathrm{B}}+m_{\mathrm{C}}\right)^{2}\right]^{1 / 4}$.

7 E.g., E. B. Wilson, Jr., J. C. Decius, and P. C. Cross, Molecular Vibrations (McGraw-Hill Book Co., New York, 1955), p. 286; e.g.,

${ }^{0} x=x(\cos \theta \cos \phi \cos \chi-\sin \phi \sin \chi)$

$$
+y(-\cos \theta \cos \phi \sin \chi-\sin \phi \cos \chi)+z \sin \theta \cos \phi,
$$

or see Eq. (A6) of this paper. 


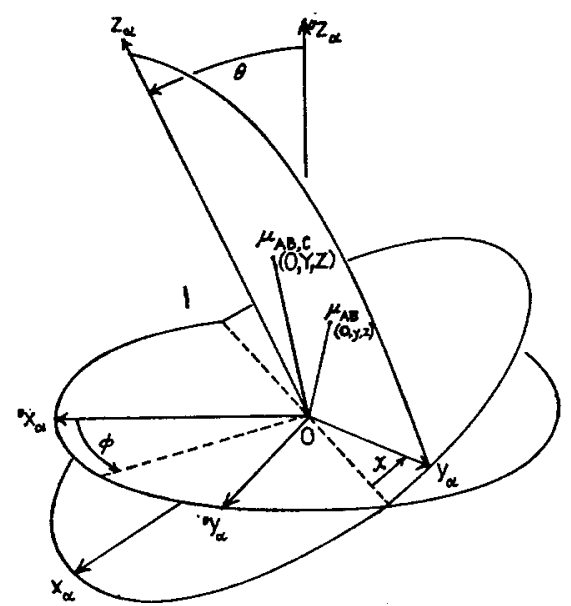

Frg. 1. Space-fixed axes $\left({ }^{0} x_{\alpha}^{0} y_{\alpha}^{0} z_{\alpha}\right)$ and body-fixed axes $\left(x_{\alpha} y_{\alpha} z_{\alpha}\right)$; oriented at Euler angles $(\theta, \phi, \chi)$. Points $(0, y, z)$ and $(0, Y, Z)$ represent the positions of reduced masses $\mu_{\mathrm{AB}}$ and $\mu_{\mathrm{AB}, \mathrm{C}}$ in massweighted space. They lie on the body-fixed $y_{\alpha} O Z_{\alpha}$ plane, which is indicated in the diagram and which corresponds to the instantaneous plane of motion of the three atoms.

A set of coordinates suited to a rearrangement collision, such as a chemical reaction, "natural collision coordinates," is next introduced. For simplicity, consider reactions for which the least unstable activated complex configuration is linear. The results can be adapted to other reactions by replacing Fig. 2 by a plot for specified nonlinear configurations and making other appropriate changes.

As in Parts I and II coordinates $n$ and $s$ are introduced to replace $z$ and $Z$, using Curve $\mathrm{C}$ in Fig. 2 . The curve is defined in terms of the potential-energy surface, Fig. 3, as discussed previously. ${ }^{1,8}$ For any point $P$ in the $(z, Z)$ subspace $n$ is the distance from $P$ to the nearest point $Q$ on Curve $\mathrm{C}$, and $s$ is the distance from an arbitrary point on $C$ to $Q$. $s$ proves to be the "reaction coordinate." The transformation $(z, Z)$ to $(n, s)$ in Fig. 2 is

$$
z=z_{Q}+n \cos \psi, \quad Z=Z_{Q}-n \sin \psi
$$

where $\left(z_{Q}, Z_{Q}\right)$ are the coordinates of $Q$ on Curve $C$.

FIG. 2. Definition of orthogonal curvilinear coordinates $n$ and $s$ of any point $P$.

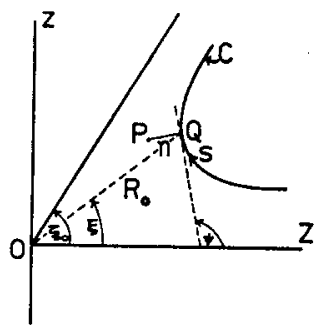

\footnotetext{
${ }^{8}$ E.g., $C$ can be the curve of least steep ascent from the reactants' valley to the potential-energy saddle point, if any, and subsequently of least steep descent to the products' valley. ${ }^{1}$ In each valley it proceeds along the bottom. An alternative choicel for $C$, as an adiabatically-vibrationally averaged path, is now somewhat more complicated because of energy loss to or gain from all the other degrees of freedom. However, a compromise choice for $C$ can be used when desired.
}

Fig. 3. Potential-energy contours for Reaction (1). Dashed line is one possible choice for Curve C in Fig. 2.

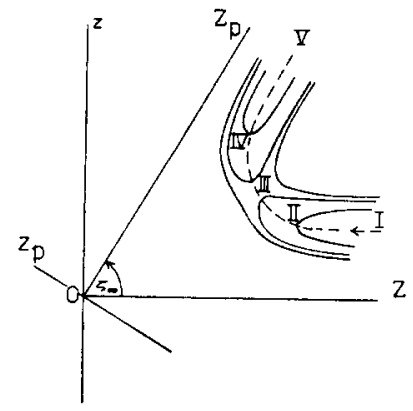

The angle $\zeta_{\infty}$ in Fig. 2 is calculated from ${ }^{5} \tan ^{2} \zeta_{\infty}=$ $m_{\mathrm{B}}\left(m_{\mathrm{A}}+m_{\mathrm{B}}+m_{\mathrm{C}}\right) / m_{\mathrm{A}} m_{\mathrm{C}}$. The half-plane of negative $Z$ is omitted in Fig. 2 to avoid double counting of configurations.

The coordinates are now $\theta, \phi, x, n, s$ and a sixth to be drawn from the $(y, Y)$ pair. The method for making this choice amounts to one for defining the orientation of the body-fixed axes in the $y_{\alpha} z_{\alpha}$ plane. We do so in a way which makes the $z_{\alpha}$ axis the line of centers of reactants when $s \cong-\infty$, the line of centers of the products when $s \cong+\infty$, and the axis of the activated complex when $s$ is in that neighborhood $\left(s=s^{\ddagger}\right)$. (The method used has several other useful properties.9a) For this purpose we introduce a local Cartesian condition at each $s$,

$$
y \sin \zeta+Y \cos \xi=0
$$

and later specify the $s$-dependent parameter $\zeta(s)$ by condition (12). The axes' orientation in the $y_{\alpha} z_{\alpha}$ plane is the one fulfilling (7) and (8) simultaneously.

The sixth coordinate $m$ is chosen to have a magnitude $\left(y^{2}+Y^{2}\right)^{1 / 2}$ and a sign defined through

$$
y=-m \cos \zeta, \quad Y=m \sin \zeta .
$$

To avoid double counting of configurations, the domain of $m$ or $\chi$ is appropriately restricted..$^{\text {gb }}$ The typical shape
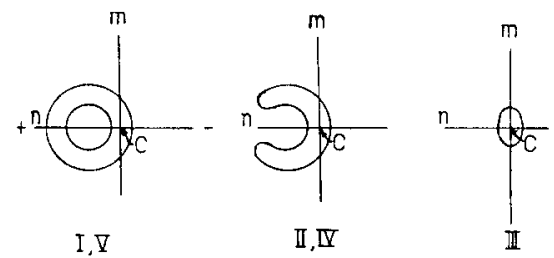

FIG, 4. Potential-energy profiles in $(m, n)$ subspace for several values of $s$ in Fig. 3 .

- (a) It gives a locally Cartesian internal coordinate space at each $s$, can be made to make the vibrational angular momentum small near $s^{\mathfrak{t}}$, and makes the only nonzero product of inertia $\Sigma_{\alpha} m_{\alpha} y_{\alpha} z_{\alpha}$ at each $s$ vanish on the average. [As an alternative, one might have chosen the axes' orientation by making the instantaneous products of inertia vanish exactly. For resulting equations based on the customary coordinates see H. Diehl, S. Flügge, A. Volkel, and A. Weiguny, Z. Physik 162, 1 (1961) and K. F. Freed and J. R. Lombardi, J. Chem. Phys. 45, 591 (1966).] (b) Reflection in the $x_{\alpha} O z_{\alpha}$ plane in Fig. 1 yields the same configuration as increase of $\chi$ by $\pi$. Double counting is avoided by setting either $m \geqslant 0$ or $0 \leqslant x \leqslant \pi$. In the case the $\gamma$ defined in (10) is restricted to $(0, \pi)$, and then only the upper half plane in Figs. 4 and 5 is used. 


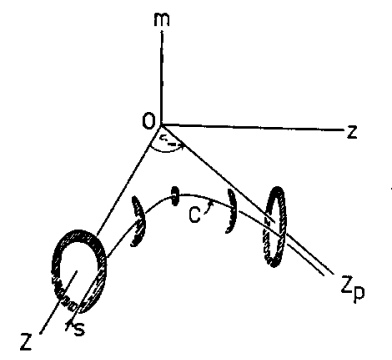

FIG. 5. The profiles in Fig. 4 are the shaded regions in this $(m, n, s)$ subspace.

of the potential-energy profiles in the $(m, n)$ subspace is given in Fig. 4 and in the $(m, n, s)$ subspace given in Fig. 5. The profiles reflect the free rotation of the diatomic molecule at large $|s|$ and the increasingly hindered rotation as the syetem moves to values of $s$ and $s^{\ddagger}$. In the latter neighborhood, the rotation becomes a bending vibration. The relation between the physical motions at large $|s|$ and the coordinates in Fig. 5 is described later.

Because of the shape of the profiles we transform $(n, m)$ into $(r, \gamma)$,

$$
n=r_{0}-r \cos \gamma, \quad m=r \sin \gamma,
$$

where $r_{0}(s)$ is determined from the shape of the profiles in Fig. 3. It is a function of $s$ and is shown in Appendix I to be the pertinent diatomic bond length when $s=+\infty$ and $-\infty$. (For symmetrical reactions $r_{0}$ may be constant.)

A vibrational coordinate $\rho$ is introduced via

$$
\rho=r_{0}-r \text {. }
$$

It remains to define $\zeta(s)$. One suitable choice makes

$$
\begin{array}{ll}
\zeta=\pi-\psi & (\text { at large }-s), \\
\zeta=\xi & \left(\text { at } s=s^{\ddagger}\right), \\
\zeta=\psi & (\text { at large }+s),
\end{array}
$$

where $\xi$ is the polar angle of $Q$ in Fig. 2, and $s^{\ddagger}$ is some preselected $s$, for example, the $s$ at or near the potentialenergy maximum along Curve $\mathrm{C}$, if any. The first and third conditions make the $z_{\alpha}$ axis the line of centers of reactants at large $-s$ and of products at large $+s$, respectively. The second condition makes the vibrational angular momentum vanish for the most stable configuration at $s=s^{\ddagger}$. Each of these results is established in Appendix I.

Imposition of (13) fulfills the first and third conditions in (12). It also fulfills the second condition when $s^{\ddagger}$ occurs at the point on Curve C in Fig. 2 closest to the origin,

$$
r_{0} \cos (\zeta-\psi)-R_{0} \sin (\zeta-\xi)=0,
$$

where $R_{0}$ and $\xi$ are the polar coordinates of point $Q$ in Fig. 2,

$$
z_{Q}=R_{0} \sin \xi, \quad Z_{Q}=R_{0} \cos \xi .
$$

A typical plot of $\psi(s), \xi(s)$ and $\zeta(s)$ is given in Fig. 6 . One set of natural collision coordinates is $(s, \rho, \theta, \phi$, $\chi, \gamma)$. A set $(s, \rho, \theta, \phi, \theta, \Phi)$ which avoids kineticenergy cross terms ${ }^{10}$ at large $\pm s$ between external and internal coordinates and is used later for boundary conditions is given in Appendix II.

\section{KINETIC ENERGY}

If $\left(\omega_{x}, \omega_{y}, \omega_{z}\right)$ denote the components of the angular momentum about the body-fixed axes and $I_{x x}, I_{x y}$, etc., denote the moments and products of inertia, the kinetic energy is ${ }^{11}$

$$
\begin{array}{r}
T=\frac{1}{2} \mu\left(\dot{y}^{2}+\dot{Y}^{2}+\dot{z}^{2}+\dot{Z}^{2}\right)+\frac{1}{2}\left(I_{x x} \omega_{x}{ }^{2}+I_{y y} \omega_{y}{ }^{2}+I_{z z} \omega_{z}{ }^{2}\right) \\
-I_{y z} \omega_{y} \omega_{z}+\Omega_{x} \omega_{x}
\end{array}
$$

where cognizance of (6) was taken, so that $I_{x z}, I_{x y}, \dot{x}$, $\dot{X}, \Omega_{y}$, and $\Omega_{z}$ all vanish, $\Omega_{x}$ being the sole nonvanishing component of the vibrational angular momentum,

$$
\Omega_{x}=\mu(y \dot{z}-z \dot{y}+Y \dot{Z}-Z Y) .
$$

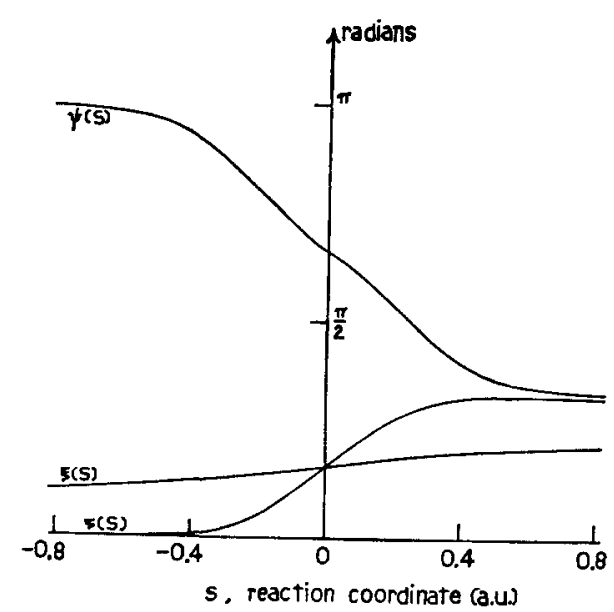

FIG. 6. Plot of $\psi(s), \xi(s)$, and $\zeta(s)$, where $s$ is the distance along Curve $\mathrm{C}$ of Figs. 2 and 3 for reaction $\mathrm{H}+\mathrm{H}_{2} \rightarrow \mathrm{H}_{2}+\mathrm{H}$. Asymptotic values are $(\pi, 0,0)$ at $s=-\infty$ and $\left(\frac{1}{3} \pi, \frac{1}{3} \pi, \frac{1}{3} \pi\right)$ at $s=+\infty$, and intercept at $s=0$ is $\left(\frac{2}{3} \pi, \frac{1}{6} \pi, \frac{1}{6} \pi\right)$, respectively. Calculations utilize potential-energy surface of M. Karplus, R. N. Porter, and R. D. Sharma, J. Chem. Phys. 43, 3259 (1963).

${ }^{10}$ These cross terms reflect the fact that the free rotation at large $\pm s$ does not obey a simple equation when expressed in coordinates relative to moving body-fixed axes but does so when its coordinates are relative to space-fixed ones. The transformation (A5) is one from $(\gamma, \chi)$ to the space-fixed coordinates $(\Theta, \Phi)$.

${ }^{11}$ The calculations parallel those in Chap. 11 of Ref. 7, noting that Eq. 6 of Sec. 11-1 there is not used; e.g., if the column vectors in (5) are denoted by ${ }^{\circ} \mathrm{r}$ and $\mathrm{r}$, and the corresponding ones involving ${ }^{\circ} X$ etc., by ${ }^{\circ} \mathbf{R}$ and $\mathbf{R}$, then

$T=\frac{1}{2} \mu\left({ }^{0} \dot{\mathbf{r}}^{T 0} \mathbf{r}+{ }^{0} \dot{\mathbf{R}}^{T 0} \dot{\mathbf{R}}\right)=\frac{1}{2} \mu \dot{\mathbf{r}}^{T} \dot{\mathbf{r}}+\frac{1}{2} \mu\left(\mathbf{r}^{T} \dot{\mathbf{S}}^{T} \mathbf{S}+\mathbf{R}^{T} \dot{\mathbf{S}} \dot{\mathbf{S}}^{T} \mathbf{R}\right)$

$+\mu\left(\mathbf{r}^{T} \dot{\mathbf{S}} \mathbf{S}^{T} \dot{\mathbf{r}}+\mathbf{R}^{T} \dot{\mathbf{S}}^{T} \dot{\mathbf{R}}\right)$,

where $\mathbf{r}^{T}$ is the transpose of $\mathbf{r}$. The first, second, and third terms are $T_{\text {vib }}, T_{\text {rot }}$, and $T_{\text {cor }} ; \mathbf{S S}^{T}$ is a $3 \times 3$ matrix whose first, second, and third rows are found to be $\left(0, \omega_{z},-\omega_{y}\right),\left(-\omega_{z}, 0, \omega_{x}\right)$ and $\left(\omega_{y},-\omega_{x}, 0\right)$. The $\dot{\mathbf{S}}^{T}$ term equals $\dot{\mathbf{S}} \mathbf{S}^{T}\left(\mathbf{S S}^{T}\right)$ and so is also readily expressed in terms of these $\omega$ 's. The cited relation between $\mathbf{S S}^{T}$ and the $\omega^{\prime}$ ' can be derived as in H. C. Corben and P. Stehle, Classical Mechanics (John Wiley \& Sons, New York, 1960), 2nd ed., Chap. 9, with obvious major changes in notation (e.g., $\omega_{z}, x$, and ${ }^{0} x$ instead of ${ }^{0} \omega_{x},{ }^{0} x$, and $x$ ) and in definition of Eulerian angles. Ours are those of Ref. 7 . 
The values of $\left(\omega_{x}, \omega_{y}, \omega_{z}\right)$ are known ${ }^{12}$ in terms of $\dot{\theta}, \dot{\phi}$, and $\dot{\chi} . I_{y z}$ is given in Ref. 13. In Appendix I $\Omega_{x}$ [and, hence, the coefficient of $\dot{\gamma} \omega_{x}$ in (18)] is shown to vanish at $s^{\ddagger}$.

In terms of the coordinates $(\rho, s, \theta, \phi, \chi, \gamma)$ one finds

$$
T=T_{0}+T_{1}
$$

where

$$
\begin{aligned}
& T_{0}=\frac{1}{2} \mu\left(\eta^{2} \dot{s}^{2}+\dot{\rho}^{2}\right)+\frac{1}{2} \mu r^{2}\left(\dot{\gamma}^{2}+\omega_{z}^{2} \sin ^{2} \gamma\right) \\
& +\frac{1}{2} I_{x x}\left(\omega_{x}^{2}+\omega_{y}^{2}\right)-\mu \dot{\gamma} \omega_{x} r^{2}\left(f-A_{m} \cos \gamma\right), \\
& T_{1}=2 \mu \dot{r} \dot{r}_{0} \sin ^{2 \frac{1}{2}} \gamma+\mu r \sin \gamma\left[\dot{r}_{0} \dot{\gamma}-\frac{1}{2} \omega_{y}{ }^{2} r \sin \gamma\right. \\
& \left.-r\left(f \cos \gamma-A_{m}\right) \omega_{y} \omega_{z}+\left(A_{s} \dot{s}+A_{m} \dot{r}\right) \omega_{x}\right],
\end{aligned}
$$

where $T_{1}$ contains terms which are small ${ }^{14}$ both at large $\pm s$ and for the stablest configurations at $s=s^{\ddagger} ; \dot{r}_{0}$ is $\left(d r_{0} / d s\right) \dot{s}, \kappa$ in (20) is the curvature of Curve $\mathrm{C}$ at any $s$, and

$$
\begin{aligned}
& f=\cos (\zeta-\psi), \quad \kappa=-d \psi / d s, \quad \kappa_{1}=-d \zeta / d s, \\
& \eta^{2}=(1+\kappa n)^{2}+\left(\kappa_{1} m\right)^{2} \text {, } \\
& A_{8}=R_{0} \kappa_{1} \cos (\zeta-\xi)-\left(1+\kappa n+\kappa_{1} n\right) \sin (\psi-\zeta) \\
& -\left(d r_{0} / d s\right) f \\
& A_{m}=\left[r_{0} \cos (\zeta-\psi)-R_{0} \sin (\zeta-\xi)\right] / r, \\
& I_{x x} \equiv \mu R^{2} \\
& =\mu\left[R_{0}^{2}-r_{0}^{2}+r^{2}+2 n\left\{R_{0} \sin (\xi-\psi)+r_{0}\right\}\right] .
\end{aligned}
$$

$\left[R\right.$ is defined as $\left(I_{x x} / \mu\right)^{1 / 2}$ and equals the distance of any point in Fig. 5 to the origin.]

Using the value ${ }^{12}$ for $\omega$ one finds

$$
\begin{array}{r}
T_{0}=\frac{1}{2} \mu\left(\eta^{2} \dot{\mathrm{s}}^{2}+\dot{\rho}^{2}\right)+\frac{1}{2}\left(\mu r^{2}\right)\left[\dot{\gamma}^{2}+(\cos \theta \dot{\varphi}+\dot{\chi})^{2} \sin ^{2} \gamma\right] \\
+\frac{1}{2}\left(\mu R^{2}\right)\left(\dot{\theta}^{2}+\sin ^{2} \theta \dot{\varphi}^{2}\right) \\
-\mu \dot{\gamma}(\sin \chi \dot{\theta}-\sin \theta \cos \chi \dot{\varphi}) r^{2}\left(f-A_{m} \cos \gamma\right) ;
\end{array}
$$

$\eta^{2}$ and $R^{2}$ in (20) and (22) can be simplified.15

In (20) $f$ vanishes for the point on Curve C closest to the origin in Fig. 2. At large $-s$ and at large $+s$, $f$ is -1 and +1 , respectively. Because of the presence of this $f$ term, the component of the angular momentum

\footnotetext{
12 Reference 7, p. 282.

${ }^{13} I_{y z}$ is $\mu(y z+Y Z)$. Use of (7), (9), and (14) yields $I_{y z}=$ $\mu m\left[R_{0} \sin (\zeta-\xi)-n \cos (\psi-\zeta)\right]$, which becomes $\mu r^{2}(f \cos \gamma-$ $\left.A_{m}\right) \sin \gamma$ on using (10) and (21b).

${ }_{14}$ At large $\pm s$ in Eq. (19), $\dot{\mathbf{r}}_{0}=0, f \cos \gamma$ vanishes in the mean, $A$, and $A_{m}$ vanish, and $\omega_{y}^{2} r^{2} \sin ^{2} \gamma$ is dominated by the $I_{x x} \omega_{y}^{2}$ in (18). At the most stable configuration for $s=s^{\ddagger}$ all terms in $T_{1}$ vanish since $\sin \gamma$ does. However, neglect of $\left(\omega_{y} \omega_{z} \sin \gamma\right)$ is justified not simply because $\sin \gamma=0$. [The $\left(\omega_{z} \sin \gamma\right)^{2}$ in $T_{0}$ has a similar factor and must be retained to avoid loss of $\chi$.] Rather, the $\omega_{\nu} \omega_{z} \sin \gamma$ can be neglected at $s^{\ddagger}$ when (12) is imposed, because the coefficient $\left(f \cos \gamma-\mathrm{A}_{m}\right)=f-f=0$ for the most stable configuration at $s^{\ddagger}$.

${ }_{15}$ E.g., $\left(\kappa_{1} m\right)^{2}=0$ both at large $\pm s$ (since $\kappa_{1}=0$ there) and for the most stable configurations near $s=s^{\ddagger}$ (since $m=0$ there). Since $n=r_{0}-r$ for the most stable configurations at $s^{\ddagger}(\gamma=0)$, while $\kappa n$ vanishes at large $\pm s$, it follows that $\eta^{2} \cong(1+\kappa \rho)^{2}$. At small $s, n \cong 0$, so $R \cong R_{0}$. At large $|s|, R_{0} \sin (\xi-\psi)=-r_{0}$. Since $r \cong r_{0}$ its equilibrium value at large $|s|$, one finds $R \cong R_{0}$ there. Therefore, at all $s$, one might take $R \cong R_{\mathrm{q}}$.
}

along the body-fixed $z_{\alpha}$ axis, $p_{x}$, is not a constant of the motion, even when the Hamiltonian is $T_{0}+V$.

The momenta conjugate to the above velocities are defined in the usual manner: $p_{i}=\partial T / \partial \dot{q}_{i}$ for the present conservative system.

\section{POTENTIAL-ENERGY FUNCTION}

Potential-energy plots were given in Figs. 3-5. In terms of $(m, Z, z)$ or, what is the same space, $(m, n, s)$ the motion of the system can be represented as one largely confined to the shaded region in Fig. 5. ${ }^{16}$ That region is annular in some places and "semiannular" in others. The potential-energy function has the form

$$
V=V_{1}(s)+V_{2}(\rho, \gamma, s) \text {, }
$$

where $V_{1}(s)$ is the value on Curve $\mathrm{C}$ and $V_{2}$ is the increment to go to any other point at the same $s$. $V_{2}$ varies mainly with $\rho$ and $\gamma$, and slowly with $s$. At large $\pm s$ it is independent of $\gamma$.

When the potential-energy coupling between the $\rho$ and $\gamma$ motions can be ignored, $V_{2}$ can be written as

$$
V_{2}(\rho, \gamma, s) \cong V_{2}(0, \gamma, s)+V_{2}(\rho, 0, s) \text {. }
$$

Since $V_{2}$ is zero on Curve $\mathrm{C}$ and since $\rho=\gamma=0$ on that curve, $V_{2}(0,0, s)$ is zero.

\section{EQUATIONS OF MOTION AND BOUNDARY CONDITIONS}

The method in Ref. 12 of conversion from $T$ to $H$ for Cartesian internal coordinates is extended to curvilinear ones in Appendix III. The Hamilton-Jacobi and Schrödinger equations are obtained in the standard way, ${ }^{17,18}$ the former by setting $p_{i}=\partial W / \partial \dot{q}_{i}$, where $W$ is Hamilton's characteristic function.

With the usual boundary conditions ${ }^{19}$ in collision problems, the wavefunction at large separation distances of the reactants is the sum of a plane wave (having reactants in their initial state) and outgoing (scattered waves) in all internal states; at large separation distances of products the wavefunction consists of outgoing (scattered) waves. The total in-going flux equals the total out-going one in the stationary-state description. These boundary conditions, expressed in terms of the usual coordinates, are readily rephrased in terms of the natural collision coordinates:

Instead of describing reactants with a different label

${ }^{16}$ In Fig. 6 it is appropriate to draw the $m$ axis as rectilinear, since the $(m, Z, z)$ subspace is almost Euclidean: The square of the distance between adjacent points is $d z^{2}+d Z^{2}+d m^{2}+\left(\kappa_{1} m\right)^{2} d s^{2}$, i.e., $\left[(1+\kappa n)^{2}+\left(\kappa_{1} m\right)^{2}\right] d s^{2}+d n^{2}+d m^{2}$. The space would be exactly Euclidean if the $\left(\kappa_{1} m\right)^{2} d s^{2}$ term were absent. Fortunately, it is small since $\kappa_{1}=0$ when $\pm s$ is large, and $m=0$ at the most stable configurations near $s=s^{\ddagger}$. The $(m, n)$ subspace in Fig. 4 is exactly Euclidean.

${ }^{17} \mathrm{H}$. Goldstein, Classical Mechanics (Addison-Wesley Publ. Co., Inc., Reading, Mass., 1950), pp. $280 \mathrm{ff}$.

${ }^{18}$ E.g., W. Pauli, Jr., in Handbuch der Physik, S. Flügge, Ed. (Springer-Verlag, Berlin, 1958), Vol. 5, p. 39.

19 E.g., A. Messiah, Quanium Mechanics (John Wiley \& Sons, Inc., New York, 1963), Vol. 2, p. 835. 
from that for products, it suffices to note that now the former corresponds to $s \rightarrow-\infty$ and the latter to $\rightarrow+\infty$. At large separation distances of reactants that distance equals $Z c, c$ being the scaling factor in (3) and $Z$ equalling $-\left(s+s_{0}\right)$, where $s_{0}$ is a known constant. ${ }^{20} \mathrm{At}$ large separation distance of products that distance is $Z_{p} c_{p}, c_{p}$ being the scaling factor in Ref. 6 and $Z_{p}$ equalling $s+s_{0}{ }^{p}$, where $s_{0}{ }^{p}$ is a known constant. ${ }^{20}$

The internal states at large $|s|$ are described in terms of coordinates $(r, \theta, \Phi)$, where $\theta$ and $\Phi$ are defined by Eq. (A4) of Appendix II. $\theta$ and $\Phi$ describe the orientation of the diatomic species with respect to the spacefixed axis; $r$ is the scaled interatomic distance in the diatomic species. In Appendix II it is shown that at large $\pm s, f \gamma$ and $\chi+\frac{1}{2} \pi$ describe the orientation of the diatomic species with respect to axes $\left(x^{\prime} y^{\prime} z^{\prime}\right)$ obtained from the space-fixed ones by a rotation $\mathbf{S}_{\theta_{\varphi}} \cdot{ }^{21}$ At large $|s|$ $\theta$ and $\varphi$ describe the orientation of the two species (Appendix II). Each rotational wavefunction, $\psi_{\mathrm{rot}}{ }^{j m_{j}}(\theta, \Phi)$ in the boundary condition at large $|s|$ is written in terms of functions of $\gamma$ and $\chi$ using $^{22}$

$\psi_{\mathrm{rot}}{ }^{j m_{j}}(\Theta, \Phi)=\sum_{\Omega=-j}^{j} \psi_{\mathrm{rot}}^{j \Omega}\left(f \gamma, \chi+\frac{1}{2} \pi\right) D_{m_{j} \Omega^{j}}{ }^{j *}(\varphi, \theta, 0)$,

where $\Omega$ is the component of $j$ along the $z^{\prime}$ axis, i.e., along the line of centers of the two species, and $D_{m_{j} \Omega}{ }^{j *}$ is a matrix element of a rotation operator $D^{+}$.

The classical mechanical problem is an initial value one, and the usual boundary conditions can also be written in terms of the present coordinates by expressing the separation distance in terms of $s$ as above. Further, when the space-fixed axes are chosen so that the ${ }^{0} z_{\alpha}$ axis is along the initial relative velocity vector and so that the initial orbital angular momentum lies along the ${ }^{0} y_{\alpha}$ axis the initial values of $\theta$ and $\varphi$ are zero. Inspection of (A4) then shows that the initial values of $f \gamma$ and $\chi+\frac{1}{2} \pi$ equal those of $\Theta$ and $\Phi$, respectively.

\section{PHYSICAL MOTIONS AT LARGE $|s|$ AND FIG. 5}

The coordinates in Fig. 5 are related to $y, Y, z, Z$. (The first two yield $m$.) Thus, this figure describes motion in the instantaneous plane formed by the three atoms, the $y_{\alpha} 0 z_{\alpha}$ plane in Fig. 1. Large $|s|$ means large separations of the reactants or products.

At large $|s|$, it is shown in Appendices I and II, $r$ is the mass-scaled instantaneous bond length of the diatomic species present (reactant at large $-s$ and prod-

\footnotetext{
${ }^{20}$ For example, let $s=0$ correspond to the point on curve $C$ nearest the origin in Fig. 2. The distance $-s$ to any point on $C$ at large negative $s$ can be compared with the corresponaing value of $Z$ for that point and $s_{0}$ determined thereby. Similar remarks apply to $s_{0}{ }^{p}$, but with $Z$ replaced by ${ }^{6} Z_{p}$ and with the distance measured to some point on $C$ at very positive $s$.

${ }^{21}$ When $S_{x \theta \phi}{ }^{T}$ in (A4) is written as $S_{\theta \phi}{ }^{T} S_{x}{ }^{T}$ and (A6) is used for $S_{\chi}$ the right side of (A4) becomes $S_{\theta \phi}{ }^{T}$ operating on a vector where components are $\left[\sin \gamma \cos \left(\chi+\frac{1}{2} \pi\right), \cos \gamma \sin \left(\chi+\frac{1}{2} \pi\right), \cos \gamma\right]$.

${ }^{82}$ D. M. Brink and G. R. Satchler, Angular Momentum (Clarendon Press, Oxford, England, 1962), pp. 21 and 26.
}

uct at large $+s)$. For any given $r$ and at large $|s|$, the representative point in Fig. 5 lies on a cylinder of axis $0 Z$ and radius $r$ (at large $-s$ ) or of axis $0 Z_{p}$ and radius $r$ (at large $+s$ ). At large $|s|$, rotation of the diatomic species with respect to the body-fixed axes corresponds to the rotation of the representative point about the relevant cylinder axis, $0 Z$ or $0 Z_{p}$, at fixed $r$. Vibration of the diatomic species at large $|s|$ corresponds to motion of the representative point normal to the surface of the relevant cylinder. Radial translational motion of the two species (reactants at large $-s$, products at large $+s$ ) corresponds to motion of the representative point parallel to the relevant cylinder axis $(0 Z$ at large $-s, 0 Z_{p}$ at large $+s$ ).

When the diatomic species at large $|s|$ has its equilibrium bond length, i.e., when $r=r_{0}$, the cited cylinder includes the relevant linear portion of curve $C$ on its surface.

\section{REACTION PATH MULTIPLICITY}

When both Reactions (1) and (2) occur, Fig. 2 is replaced by Fig. 7, with the half-plane of negative $Z$ again omitted to avoid double counting of configurations. The cross-sectional potential-energy profile for region $I$ is unaltered, but the profile near the entrance of II has two separated semiannular regions, one containing Curve $\mathrm{C}$ and the other containing Curve $\mathrm{G}$. The former leads to Reaction (1) and the latter to Reaction (2). From this point on, each of these two reaction paths is described in terms of its own set of natural collision coordinates, and in solving the Schrödinger equation account should be taken of this "bifurcation" of the wavefunction.

\section{REACTION IN A PLANE}

The simplest system which provides information on the vibrational-translational changes during a reactive collision is reaction in a line. The simplest system providing information on the conversion of rotation into bending vibrations and then into product rotations, with orbital and translational coupling included, is reaction in a plane. The motion occurs in the ${ }^{0} x_{\alpha}^{0} y_{\alpha}$ plane when one sets $\theta=\pi / 2$, and $\chi=0$, for then ${ }^{0} z_{\alpha}=-x_{\alpha}=0$ according to (5) and (6). We now have, $\omega_{y}=\omega_{z}=0$,

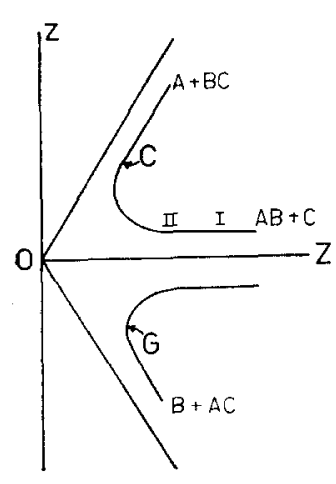

FIG. 7. Counterpart of Fig. 2 for Reactions (1) plus (2). 
and $\omega_{x}=-\dot{\phi}^{23}$ Thus, (18) and (19) become ${ }^{24}$

$T_{0}=\frac{1}{2} \mu\left(\eta^{2} \dot{s}^{2}+\dot{\rho}^{2}\right)+\frac{1}{2} \mu r^{2} \dot{\gamma}^{2}+\frac{1}{2} \mu R^{2} \dot{\phi}^{2}+\mu \dot{\gamma} \dot{\phi} r^{2}\left(f-A_{m} \cos \gamma\right)$,

$T_{1}=2 \mu \dot{r}_{0} r \sin ^{2 \frac{1}{2}} \gamma+\mu r \sin \gamma\left[\dot{r}_{0} \dot{\gamma}-\dot{\phi}\left(A_{s} \dot{s}+A_{m} \dot{r}\right)\right]$.

Now, $\Phi=\phi+f \gamma$ and $\theta=0$.

\section{DISCUSSION ON BIMOLECULAR REACTIONS}

The physical nature of the various coordinates at $s= \pm \infty$ was considered in a previous section. In the activated complex region $\left(s=s^{\ddagger}\right) \rho$ and $s$ describe the symmetric stretching vibration and the asymmetric (imaginary frequency) one, respectively; when (12) is imposed, $\theta$ and $\phi$ describe the orientation of the activated complex and $\gamma$ and $\chi$ describe its doubly degenerate bending vibration. In intermediate regions of $s$, the latter coordinates describe a hindered rotation coupled rather than a free rotation or a bending vibration.

One approximate solution, the nearly vibrationally adiabatic one, was discussed briefly in a previous paper, ${ }^{4}$ together with some implications for activated complex theory and related topics. A vibrationally adiabatic reactive collision can be regarded as the chemical analog of the elastic collision in physical scattering, and both can serve as a basis for more detailed calculations. The present coordinates provide a vehicle for treating this vibrationally adiabatic chemical reaction. The latter will be given in a later paper, together with a calculation of nonadiabatic corrections.

\section{ACKNOWLEDGMENTS}

It is a particular pleasure to acknowledge the warm hospitality of Professor Hirschfelder, Professor Bernstein, and their colleagues while the writer was a Visiting Professor at the Theoretical Chemistry Institute of the University of Wisconsin. Mr. Shiou-Fu Wu, who is currently working on applications of the present work, has also made a number of helpful suggestions and provided Fig. 6.

\section{APPENDIX I. CONSEQUENCES OF EQ.}

We first show that at large $-s \mathrm{Eq}$. (12) yields $X=Y=0$, so that the line of centers of reactants is along the $z_{\alpha}$ axis, and that at large $+s$ Eq. (12) yields $X_{p}=Y_{p}=0$, so that the line of centers of the products is along the $z_{\alpha}$ axis. These results are embodied in the second half of (A1) and (A2). The first half of (A1) and (A2) is derived for use in Appendix II.

At large $-s$, use of Eqs. (6), (9), (10), (12), (14) and of the fact that $R_{0} \sin \xi$ and $\zeta$ now equal $r_{0}$ and 0

\footnotetext{
23 Compare the equations in Ref. 11 when $\theta=\frac{1}{2} \pi$ and $\chi=0$.

24 From (28) and (29) the kinetic energy in Part I for collision on a line can be readily obtained, using the fact that on a line,
$\phi=\gamma=0$, so $n=\rho$.
}

show that

$$
\begin{aligned}
& \left(\begin{array}{l}
x \\
y \\
z
\end{array}\right)=\left(\begin{array}{c}
0 \\
-r \sin \gamma \\
r \cos \gamma
\end{array}\right) ; \\
& \left(\begin{array}{c}
X \\
Y \\
Z
\end{array}\right)=\left(\begin{array}{l}
0 \\
0 \\
Z
\end{array}\right) \quad(\text { large }-s) .
\end{aligned}
$$

Thus, $Z$ is now the mass-scaled distance between the mass centers of the two reactants, since the other two components of that radius vector vanish.

At large $+s$ use of similar equations and of the fact that $R_{0} \sin \left(\zeta_{\infty}-\xi\right)$ and $\zeta$ now equal $r_{0}$ and $\zeta_{\infty}$ show that

$$
\begin{gathered}
\left(\begin{array}{c}
x_{p} \\
y_{p} \\
z_{p}
\end{array}\right)=-\left(\begin{array}{c}
0 \\
r \sin \gamma \\
r \cos \gamma
\end{array}\right) ; \\
\left(\begin{array}{c}
X_{p} \\
Y_{p} \\
Z_{p}
\end{array}\right)=\left(\begin{array}{l}
0 \\
0 \\
Z_{p}
\end{array}\right) \quad(\text { large }+s) .
\end{gathered}
$$

Thus, $Z_{p}$ is now seen to equal the mass-scaled distance between the mass centers of the two products.

The vibrational-angular-momentum component $\Omega_{x}$ defined by (16) is found to equal

$$
\Omega_{x}=\mu\left(A_{\varepsilon} \sin \gamma \dot{s}+A_{m} \dot{m}-r \dot{\gamma}\right) r .
$$

The most stable configuration at $s=s^{\ddagger}$ occurs at $\gamma=0, r=r_{0}$, and so one finds $A_{s} \sin \gamma=0$ at this point. Also at this point, if (12) is imposed, the last two terms in (A3) cancel each other. Thus, the choice $\zeta=\xi$ in (12) causes $\Omega_{x}$ to vanish for this stablest configuration at $s^{\ddagger}$ and is equivalent to employing an Eckart condition $^{25}$ at $s=s^{\ddagger}$, a condition hitherto employed only for stable molecules.

\section{APPENDIX II. RELATION OF $\theta, \Phi$ TO $\gamma, \chi$ AND PROPERTIES}

The body-fixed coordinates of the diatomic reactant $\mathrm{AB}$ are $(x, y, z)$. Thus, (A1) shows that at large $-s$ the mass-scaled radius vector joining $A$ to $B$ has a length $r$ and makes an angle $-\gamma$ with the (body-fixed) $z_{\alpha}$ axis. The body-fixed coordinates of the diatomic product $\mathrm{BC}$ are $\left(x_{p}, y_{p}, z_{p}\right)$. Hence, (A2) reveals that at large $+s$ the mass-scaled radius vector joining $\mathrm{B}$ to $C$ has a length $r$ and makes an angle $+\gamma$ with the $z_{\alpha}$ axis. Since $f$, which equals $\cos (\zeta-\psi)$, equals -1 at

${ }^{25}$ C. E. Eckart, Phys. Rev. 47, 552 (1935). 
large $-s$ and +1 at large $+s$, the above angle could be written in each case as $f \gamma$.

It is useful to define a set of coordinates $\theta, \Phi$, via (A4),

$$
\left(\begin{array}{c}
\sin \theta \cos \Phi \\
\sin \theta \sin \Phi \\
\cos \theta
\end{array}\right)=\mathbf{S}_{\chi \theta^{\theta}}{ }^{T}\left(\begin{array}{c}
0 \\
\sin f \gamma \\
\cos f \gamma
\end{array}\right) \text {. }
$$

Comparison of (5), (A1), and (A4) shows that at large $-s$ the spaced-fixed coordinates of the line joining $\mathrm{A}$ to $\mathrm{B}$ and those of the line joining the mass center of $\mathrm{AB}$ to atom $\mathrm{C}$, namely $\left({ }^{0} x,{ }^{0} y,{ }^{0} z\right)$ and $\left({ }^{0} X,{ }^{0} Y,{ }^{0} Z\right)$, respectively, can be written in terms of polar coordinates $(r, \theta, \Phi)$ and $(Z, \theta, \phi)$, respectively, in the standard way (e.g., ${ }^{0} x=r \cos \theta \sin \Phi, \cdots,{ }^{0} Z=Z \cos \theta$ ). Use of this result and Eq. (4) shows that there are no cross terms in the kinetic energy, when the latter is expressed in terms of $(r, Z, \theta, \Phi, \theta, \phi)$ [i.e., of $(s, \rho, \theta, \Phi, \theta, \phi)$ since, at large $-s, r$ is a function of $\rho$ only and $Z$ is a function of $s$ only].

Similarly, at large $+s$, a comparison of (5), (A2), and (A4) shows that the space-fixed coordinates of the line joining $B$ to $C$ and those of the line joining $A$ to the mass center of $\mathrm{BC}$, namely $\left(-{ }^{0} x_{p},-{ }^{0} y_{p},-{ }^{0} z_{p}\right)$ and $\left({ }^{0} X_{p},{ }^{0} Y_{p},{ }^{0} Z_{p}\right)$, respectively, can be written in terms of polar coordinates $(r, \theta, \Phi)$ and $\left(Z_{p}, \theta, \phi\right)$, respectively (e.g., $\left.-{ }^{0} x_{p}=r \cos \theta \sin \Phi, \cdots,{ }^{0} Z_{p}=Z_{p} \cos \theta\right)$. Since the kinetic energy (4) can be expressed as the sum of squares of ${ }^{0} x_{p}, \cdots,{ }^{0} Z_{p}$ by a rotation through an angle $\zeta_{\infty}$ in each subspace $\left({ }^{0} x,{ }^{0} X\right),\left({ }^{0} y,{ }^{0} Y\right),\left({ }^{0} z,{ }^{0} Z\right)$, and since one can now introduce the polar coordinates just cited, there are no cross terms at large $|s|$ in a kinetic energy expressed in terms of $\left(r, Z_{p}, \Theta, \Phi, \theta, \phi\right)$, i.e., of $(s, \rho, \theta, \Phi, \theta, \phi)$.

The coordinates $\chi$ and $\gamma$ can be expressed in terms of $\theta, \Phi$, by application of the rotational matrix $\mathbf{S}_{\theta \phi}$ to both sides of (A4),

$$
\begin{aligned}
\left(\begin{array}{c}
-\sin \chi \sin f \gamma \\
\cos \chi \sin f \gamma \\
\cos f \gamma
\end{array}\right) \\
\quad=\left(\begin{array}{c}
\cos \theta \sin \theta \cos (\Phi-\phi)-\sin \theta \cos \theta \\
\sin \theta \sin (\Phi-\phi) \\
\sin \theta \sin \theta \cos (\Phi-\phi)+\cos \theta \cos \theta
\end{array}\right),
\end{aligned}
$$

where we note that $\mathbf{S}_{\theta \phi}$ is $\mathbf{S}_{\theta} \mathbf{S}_{\phi}, \mathbf{S}_{\chi \theta \phi}$ is $\mathbf{S}_{\chi} \mathbf{S}_{\theta} \mathbf{S}_{\phi}, T$ denotes transpose, and

$$
\mathbf{S}_{\theta}=\left(\begin{array}{ccc}
\cos \theta & 0 & -\sin \theta \\
0 & 1 & 0 \\
\sin \theta & 0 & \cos \theta
\end{array}\right),
$$

$$
\begin{aligned}
& \mathbf{S}_{\phi}=\left(\begin{array}{ccc}
\cos \phi & \sin \phi & 0 \\
-\sin \phi & \cos \phi & 0 \\
0 & 0 & 1
\end{array}\right), \\
& \mathbf{S}_{\chi}=\left(\begin{array}{ccc}
\cos \chi & \sin \chi & 0 \\
-\sin \chi & \cos \chi & 0 \\
0 & 0 & 1
\end{array}\right) .
\end{aligned}
$$

\section{APPENDIX III. KINETIC ENERGY IN TERMS OF VARIOUS MOMENTA}

The method used with Cartesian internal coordinates for transforming $T\left(q^{i}, \dot{q}^{i}, \omega_{\alpha}\right)$ into $T\left(q^{i}, p_{i}, M_{\alpha}-\Omega_{\alpha}\right)$ in Ref. 12 can be extended to curvilinear ones as follows. We do not impose any restriction that the internal coordinate space be Euclidean. We employ in (A7)(A13) the Einstein summation convention (summation of repeated indices). Let

$$
T=\frac{1}{2} g_{i j} \dot{q}^{i} \dot{q}^{j}+\frac{1}{2} \mathfrak{g}_{\alpha \beta} \omega_{\alpha} \omega_{\beta}+\mathfrak{C}_{\alpha i} \omega_{\alpha} \dot{q}^{2}
$$

where $\alpha$ is $x, y, z$ and where $\mathscr{I}_{\alpha \beta}$ equals $I_{\alpha \alpha}$ when $\alpha=\beta$ and equals $-I_{\alpha \beta}$ when $\alpha \neq \beta$.

$\partial T / \partial \omega_{\alpha}$ is denoted by $M_{\alpha}$, and a symbol $\Omega_{\alpha}$ is defined:

$$
\Omega_{\alpha}=\mathfrak{e}_{\alpha i} g^{i j} p_{j}
$$

where $g^{i j}$ is conjugate to $g_{i j}\left(g^{i k} g_{k j}=g_{i k} g^{k j}=\delta_{i j}\right)$. Manipulation shows that

where

$$
M_{\alpha}-\Omega_{\alpha}=\mathfrak{g}_{\alpha} \beta^{\prime} \omega_{\beta},
$$

$$
\mathfrak{I}_{\alpha \beta}{ }^{\prime}=\mathfrak{I}_{\alpha \beta}-\mathfrak{C}_{\alpha i} g^{i j} \mathfrak{C}_{\beta j} .
$$

Let $\mu^{\alpha \beta}$ be conjugate to $\mathscr{I}_{\alpha \beta}{ }^{\prime}$. Inversion of (A9) thus yields

$$
\omega_{\alpha}=\mu^{\alpha \beta}\left(M_{\beta}-\Omega_{\beta}\right) .
$$

If $\Delta$ is the $3 \times 3$ determinant of the $\mathscr{I}_{\alpha \beta}{ }^{\prime \prime} \mathrm{s}, \mu^{\alpha \beta}$ equals the cofactor of $\mathcal{I}_{\alpha \beta}{ }^{\prime}$ in $\Delta$, divided by $\Delta$.

Since $T$ is a homogeneous, quadratic function of the $\dot{q}^{i}$ 's and $\omega_{\alpha}$ 's, and since $M_{\alpha}$ and $p_{i}$ are $\partial T / \partial \omega_{\alpha}$ and $\partial T / \partial \dot{q}_{i}$, Euler's theorem yields

$$
T=\frac{1}{2} p_{i} \dot{q}^{i}+\frac{1}{2} M_{\alpha} \omega_{\alpha}
$$

Subsequent manipulation, based on the expressions for $p_{i}$ and $M_{\alpha}$, yields

$T=\frac{1}{2} \sum_{i, j} g^{i j} p_{i} p_{j}+\frac{1}{2} \sum_{\alpha, \beta} \mu^{\alpha \beta}\left(M_{\alpha}-\Omega_{\alpha}\right)\left(M_{\beta}-\Omega_{\beta}\right)$.

The relation between the $M_{\alpha}$ 's and momenta conjugate to the Eulerian angles is the same as in Ref. 12. Again, the significance of $\Omega_{\alpha}$ is similar to that in Ref. 12, where it is denoted by $m_{\alpha}$ : it is the $\alpha$ th component of the "vibrational" angular momentum, for $M_{\alpha}$ is the $\alpha$ th component of the total angular momentum and Eq. (A9) shows that $M_{\alpha}-\Omega_{\alpha}$ is associated only with the rotation of the axes. 